\title{
Control of FWA gene silencing in Arabidopsis thaliana by SINE-related direct repeats
}

\author{
Yuki Kinoshita ${ }^{1}$, Hidetoshi Saze ${ }^{1}$, Tetsu Kinoshita ${ }^{1}$, Asuka Miura', Wim J.J. Soppe ${ }^{2}$, Maarten Koornneef ${ }^{2}$ and Tetsuji \\ Kakutani ${ }^{1, *}$ \\ ${ }^{1}$ Department of Integrated Genetics, National Institute of Genetics, Yata 1111, Mishima, Shizuoka 411-8540, Japan, and \\ ${ }^{2}$ Max-Planck-Institut für Züchtungsforschung, Köln, Germany
}

Received 3 August 2006; accepted 24 August 2006.

*For correspondence (fax +8155981 6804; e-mail tkakutan@lab.nig.ac.jp).

\begin{abstract}
Summary
A unique feature of late-flowering fwa epigenetic mutations is that the phenotype is caused by ectopic expression of the homeobox gene FWA. During normal development the FWA gene is expressed specifically in the endosperm in an imprinted manner. Ectopic FWA expression and disruption of imprinting can be induced in mutants of a CG methyltransferase MET1 (methyltransferase 1) or a chromatin-remodeling gene DDM1 (decrease in DNA methylation 1), suggesting that the proper FWA expression depends on cytosine methylation. However, critical methylated residues controlling FWA silencing are not pinpointed. Nor is it understood how the FWA gene is initially methylated and silenced in wild-type plants. Here we mapped sequences critical for FWA silencing by application of RdDM (RNA-directed DNA methylation) to a ddm1induced stable fwa epiallele. Transcription of double-stranded RNA corresponding to the tandem direct repeats around the FWA transcription start site induced de novo DNA methylation, transcriptional suppression and phenotypic reversion. The induced changes were heritable even without the transgene, which correlates with inheritance of CG methylation in the direct repeats. The newly silenced FWA allele was transcribed in an endosperm-specific and imprinted manner, as is the case for the wild-type FWA gene. The results indicate that methylation of the direct repeats, which presumably originated from a short interspersed nuclear element (SINE), is sufficient to induce proper epigenetic control of the FWA gene.
\end{abstract}

Keywords: DNA methylation, epigenetic, transposon, imprinting, RNA-directed DNA methylation.

\section{Introduction}

DNA methylation is one of the major heritable epigenetic marks that mediate silencing of sequences such as transposable elements, developmentally regulated genes and imprinted genes (Chan et al., 2005; Rangwala and Richards, 2004; Scott and Spielman, 2004). Most of the imprinted genes in mouse are under control of DNA methylation, as their imprinting is abolished by mutations in DNA methyltransferases (Kaneda et al., 2004; Li et al., 1993). DNA methylation is also involved in imprinting in flowering plants. In Arabidopsis two imprinted genes, MEDEA and FWA, have been identified (Grossniklaus et al., 1998; Kinoshita et al., 1999, 2004; Soppe et al., 2000; Vielle-Calzada et al., 1999). Their silencing is affected by mutations in the maintenance DNA methyltransferase MET1 (METHYLTRANSFERASE 1) or in the chromatin-remodeling gene DDM1 (decrease in DNA methylation 1; Kinoshita et al.,
2004; Vielle-Calzada et al., 1999; Xiao et al., 2003; T. Kinoshita and T. Kakutani, unpublished data), indicating that DNA methylation is also involved in imprinting of these genes. The silent state is default for these genes, and the imprinting is controlled by a 'one-way' activation that depends on the DNA demethylase gene DEMETER (Choi et al., 2002; Gehring et al., 2006; Kinoshita et al., 2004).

The approach using DNA-methylation mutants revealed that changes in DNA methylation affect imprinting. However, the critical methylated elements mediating imprinting have not been identified for any of the imprinted Arabidopsis genes (Spillane et al., 2004). Identification of such critical elements would help explain how the methylationdependent imprinting systems evolved convergently in plants and mammals. In addition, the element identification is essential to understand the mechanism controlling 
the plant-specific gene activation by DEMETER demethylase.

The FWA gene was originally identified as the gene responsible for the late-flowering phenotype of the fwa-1 and fwa-2 mutants (Koornneef et al., 1991). Genetic and molecular analysis revealed that the late-flowering phenotype was not caused by a change in nucleotide sequence, but was due to ectopic expression of the FWA gene in the background of reduced DNA methylation (Kakutani, 1997; Soppe et al., 2000). In wild-type plants, although the FWA gene is silent in adult tissue, it is specifically expressed in endosperm (Kinoshita et al., 2004). In both the epigenetic mutants and endosperm, a correlation was found between FWA transcription and loss of methylation in tandem repeats around the transcription start site (Kinoshita et al., 2004; Soppe et al., 2000). However, there has been no evidence that methylation of this specific region is causing the FWA silencing. The met1 as well as $d d m 1$ mutations affect methylation across the genome. Not only the tandem repeats, but also large chromosomal regions around them, which can be several megabases long, were hypomethylated in the met1, ddm 1 and epigenetic fwa mutants (Soppe et al., 2000).

A transgene containing the upstream region of FWA often mimics the endogenous FWA copy in expression pattern (Figure S1; Kinoshita et al., 2004). Similarly, extra copies of FWA become silenced after Agrobacterium-mediated transformation (Soppe et al., 2000). Silencing of the FWA transgene depends on the de novo methyltransferase DRM, RNA polymerase IV subunit (SDE4/NRPD1a), and genes related to small RNA-processing machinery such as RNA DEPENDENT RNA POLYMERASE 2, DICER-LIKE3, and ARGONAUTE4 (Cao and Jacobsen, 2002; Chan et al., 2004). As small RNA is generally made from double-stranded RNA, it is mysteri- ous how the FWA transgene, which does not contain an inverted repeat, could become a target of silencing mediated by a small RNA.

In this report, using a stable $d d m$ 1-induced epigenetic FWA line and the RNA-dependent DNA methylation (RdDM) strategy (Mette et al., 2000; Wesley et al., 2001), we show directly that the critical cis-elements are the tandem direct repeats around the transcription start site. The silent state of FWA was inherited even after the RdDM construct was segregated apart. Inheritance of the silent state in these plants is also associated with inheritance of CG methylation in the direct repeats. Epigenetic controls and the biological significance of the direct repeats are discussed.

\section{Results}

Stable inheritance of a ddm1-induced late-flowering trait

When an inverted repeat sequence was transcribed from a transgene, de novo methylation was induced for the same sequence (Mette et al., 2000). We applied such an RNAdirected DNA methylation (RdDM) strategy to examine directly the critical methylated sequence that silences FWA.

For the material to be silenced by RdDM, we first generated a stable epigenetic FWA line induced by the $d d m 1$ mutation. Late-flowering traits were frequently induced in self-pollinated progeny of the $d d m 1$ mutant (Kakutani, 1997; Kakutani et al., 1996). The $d d m 1$-induced late-flowering trait co-segregated with the FWA locus irrespective of the presence of the wild-type DDM1 copy (Kakutani, 1997). To make the background reasonably clean, we back-crossed the late-flowering $d d m 1$ line three times to parental wild-type Columbia plants. The late-flowering trait was inherited in a dominant manner through the back-crossing and subsequent self-pollination (a)

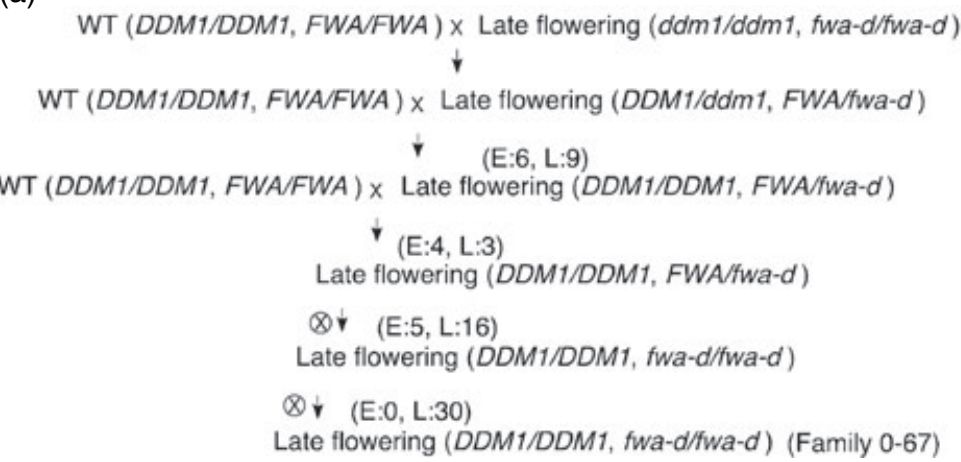

(b)
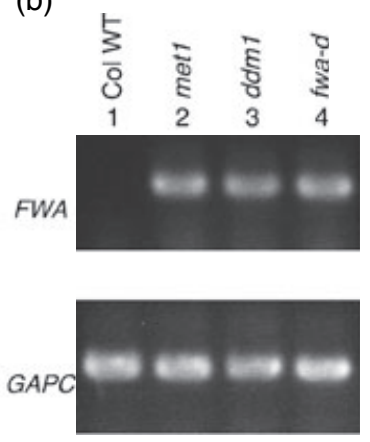

Figure 1. A $d d m$ 1-induced stable epigenetic late-flowering line.

(a) Back-crossing procedure of a late-flowering FWA allele induced by the ddm 1 mutation. The late-flowering epigenetic FWA allele ( $f w a-d$ ) was induced by selfpollinating a $d d m 1$ mutant seven times (Kakutani, 1997; Kakutani et al., 1996). WT, wild-type Columbia plant with wild-type FWA and DDM1 alleles. Segregation of late-flowering (L) and normal flowering (E) plants from each cross is shown by number of plants. The family (0-67) homozygous for the $d d m$ 1-induced fwa-d epiallele was used for the subsequent experiments. $\mathrm{X}$ and circled $\mathrm{X}$ indicate crossing and self-pollination, respectively.

(b) The late-flowering trait, heritable in the presence of the DDM1 gene, was associated with ectopic FWA expression; fwa-d represents self-pollinated progeny of family 0-67 (the last generation in a). Accumulation of FWA transcript was detected by RT-PCR. Glyceraldehyde-3-phosphate dehydrogenase (GAPC) was used as the positive control. 
procedures (Figure 1a). This phenotype was maintained even in the presence of the wild-type DDM1 allele. The late-flowering phenotype, with ectopic FWA transcription, was inherited for five generations in the DDM1/-background (Figure 1a,b). Further analyses confirmed that the lateflowering trait and the associated ectopic FWA expression of this line ( $f w a-d$ ) are stable and genetically linked to the FWA locus (Figures S2 and S3).

De novo silencing of the FWA epiallele by a transgene covering direct repeats

In order to identify the critical methylated cis-elements mediating FWA silencing, we used the stable epigenetic line (fwa-d) and RdDM strategy. For the RdDM procedure, we tested transgenes with inverted repeats covering the $5^{\prime}$ upstream region (construct $A$, Figure $2 a$ ), direct repeats around the transcription start site (construct $B$ ), and the first intron region (construct $C$ ). Transgenic plants hemizygous for the transgene were crossed to the $d d m$ 1-induced late-flowering line (fwa-d in Figure 1). Phenotypic reversion occurred in plants with the transgene containing the direct repeat around the transcription start site (construct $B$, Figure $2 b$ ). On the other hand, the transgenes containing upstream (construct $\mathrm{A}$ ) or downstream (construct $\mathrm{C}$ ) regions did not affect the late-flowering phenotype (Figure 2b). Both the constructs with the upstream and downstream half of construct $B$ (constructs $B 1$ and $B 2$ ) were effective, although the effects tend to be weaker than the construct covering the entire repeats (Figure $2 b$, right panel). These observations suggest that methylation around the direct repeats is critical for FWA silencing. All the $F_{1}$ plants without the transgene remained late flowering (Figure 2), confirming that suppression of the late-flowering phenotype was induced by the transgene. The phenotypic reversion was correlated with silencing of the FWA gene (Figure 2c)

Transgene-induced de novo silencing of the FWA epiallele was heritable and associated with CG methylation and small RNA

In addition to the crossing experiments, we directly transformed the $d d m$ 1-induced late-flowering line fwa- $d$ with the inverted repeat transgenes. The reversion from the late-

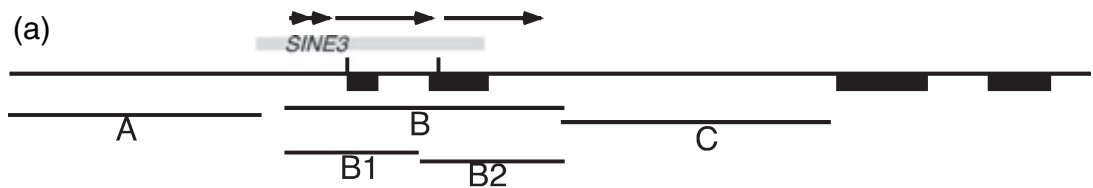

(b)

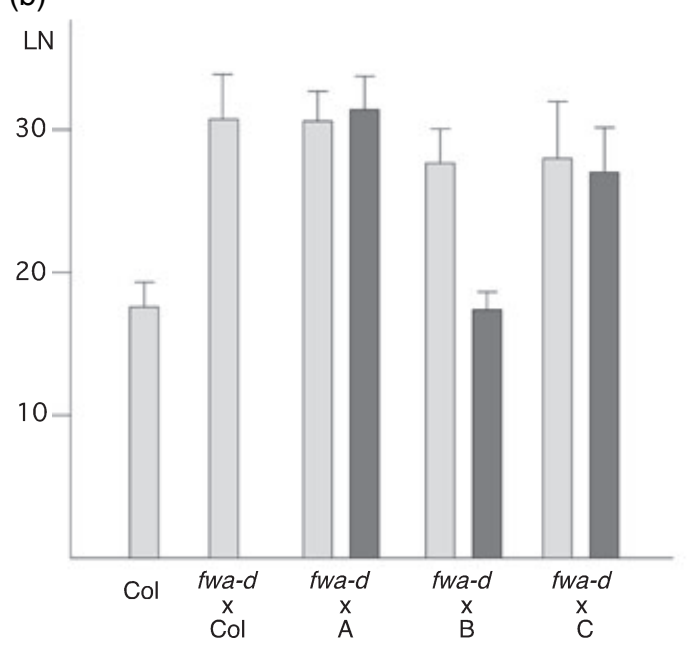

Transgene Minus
Figure 2. Reversion from the $d d m$ 1-induced late-flowering trait induced by inverted repeat transgenes.

(a) A, B, C, B1 and B2 indicate regions covered by the inverted-repeat constructs. Black boxes indicate the first four FWA exons (Soppe et al., 2000); arrows, direct repeats around the transcription start site; grey bars, regions with characteristics of the SINE insertion (Lippman et al., 2004).

(b) The fwa- $d$ was crossed to plants hemizygous for the transgenes. Bars represent standard error. Leaf numbers of six to 14 plants were examined for each class with the transgene, while two to nine plants were examined for each class without the transgene.

(c) Transcriptional silencing of FWA by the RdDM procedure.

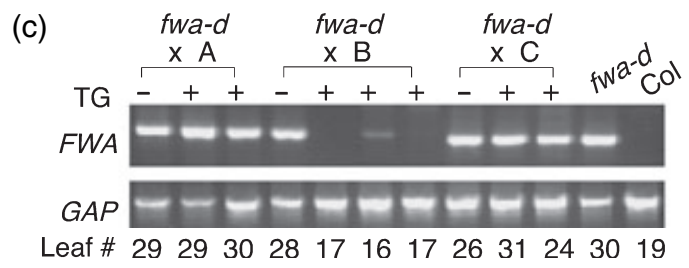


Figure 3. Inheritance of induced transcriptional FWA silencing and DNA methylation.

(a) Self-pollinated progeny of a homozygous fwa-d line transformed with construct $B$. Transformation with construct $B$ induced reversion of the late-flowering phenotype. Plants with and without the transgene were selected by PCR (top panel) from the self-pollinated progeny of such a transformant and their FWA transcription was analysed by RT-PCR. In both classes, FWA transcription remained suppressed, in contrast to the original fwa-d line (first lane).

(b) Methylation of the tandem repeats was compared. Ten to 12 clones were examined for each sample; the proportion of methylated clones is shown for each site. Red, blue and black bars represent CG, CNG and asymmetrical sites, respectively.

(c) Proportions of methylation from (b). In plants with the inverted repeat transgene, methylation of both CG and non-CG sites was induced. Methylation, especially in CG sites, was transmitted to the progeny without the transgene, resulting in a methylation pattern similar to that of the wild-type plant. Bisulfite sequencing of the upstream and downstream regions (regions $A$ and $C$ in Figure $2 a$ ) revealed that the $\operatorname{RdDM}$ transgene covering the direct repeats did not induce spread of methylation (results not shown). In addition, a gene adjacent to FWA, which is hypomethylated in the fwa-d line, remained hypomethylated in the plant family used here (Figure S5).

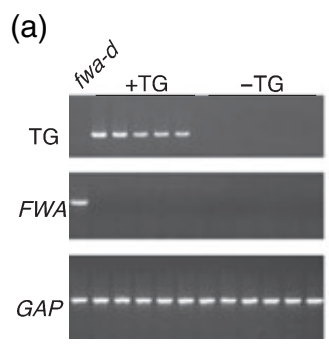

(c)

(b)
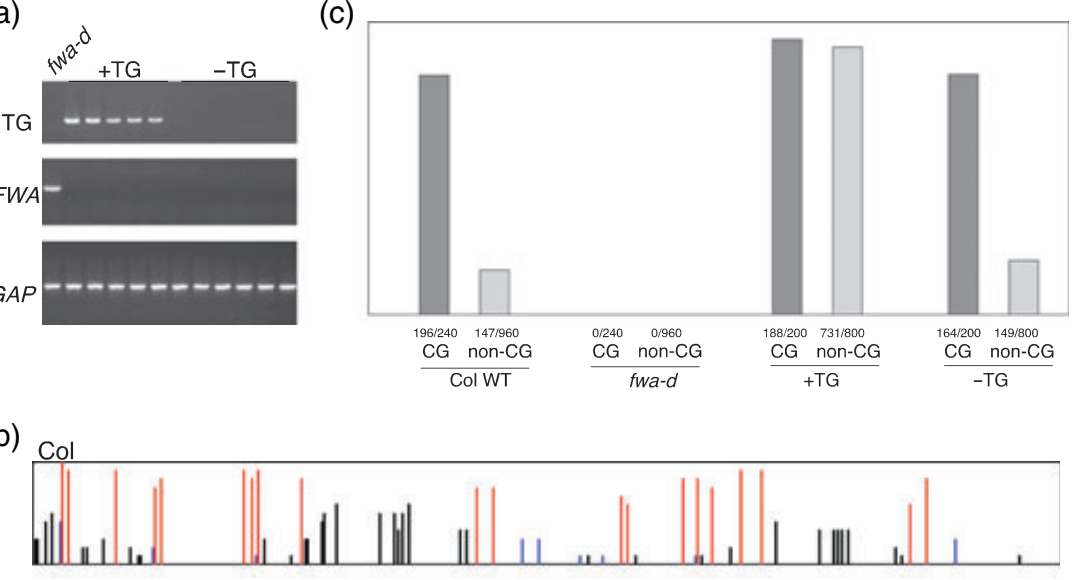

fwa-d
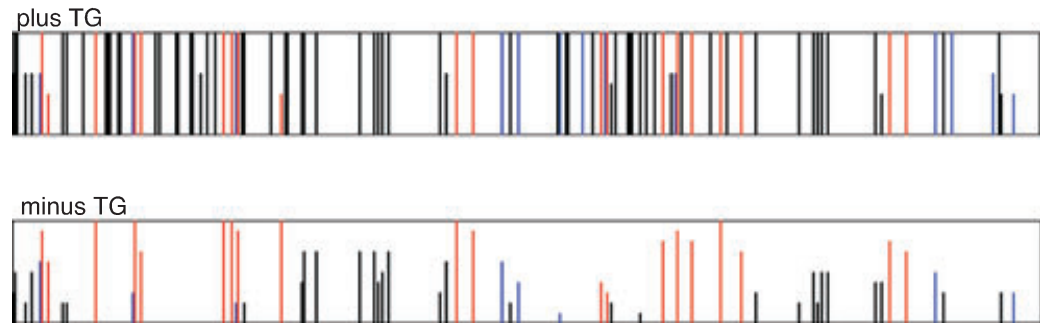

flowering phenotype was induced in all four independent lines transformed with construct B. On the other hand, neither construct $A$ or $C$ induced the reversion. Using this system, we examined the inheritance of the silencing effect over generations. In the self-pollinated progeny of a plant with re-silenced FWA locus, plants with and without the transgene segregated. Interestingly, the FWA gene was silent in both (Figure 3a), suggesting that the effect of the inverted repeat transgene was heritable. These observations suggest that a heritable chromatin-based mechanism, rather than post-transcriptional mRNA degradation, was responsible for the transgene-induced silencing (Hirochika et al., 2000; Vaucheret et al., 1998).

We examined the methylation status of the target regions in those plants by bisulfite sequencing and found that the transcriptional silencing was correlated with de novo DNA methylation (Figure 3b,c). In the wild type, cytosines in the direct repeats were methylated mainly in CG sites. On the other hand, both CG and non-CG methylation were induced by the inverted repeat transgene. In the self-pollinated progeny without the transgene, methylation at CG sites was inherited, while at non-CG sites inheritance was less efficient (Figure $3 b, c$ ). Still, FWA expression was suppressed and an early-flowering phenotype was observed in those plants (Figure 3a). These observations are consistent with the conclusion that CG methylation in the direct repeats is the heritable epigenetic mark required to silence FWA.

For the FWA direct repeats, a low level of small RNAs has been detected in the wild type (Lippman et al., 2004). A large amount of small RNA was detectable in the fwa-d plants with the inverted repeat transgene, which is the expected product from double-stranded RNA (Figure S4, lane 3). After segregating out of the RdDM transgene, the small RNA was detectable at a level comparable with that of the wild type (Figure S4, lane 4). Small RNA has generally been observed in transcriptionally repressed sequences, many of which are structurally related to transposons (Lippman et al., 2004).

\section{After RdDM of direct repeats the fwa epiallele showed a proper developmental gene-expression pattern}

During normal development in wild-type plants, the FWA gene is silent as a default state, and is derepressed in the central cell within the female gametophyte, resulting in imprinted expression in the endosperm (Kinoshita et al., 2004). In order to see whether the de novo silencing induced by the double-stranded RNA was derepressed in endosperm, we examined RNA in endosperm. The FWA transcript was detected in the endosperm even after de novo silencing by the RdDM construct (Figure 4a, left panel). As in the original 
(a)
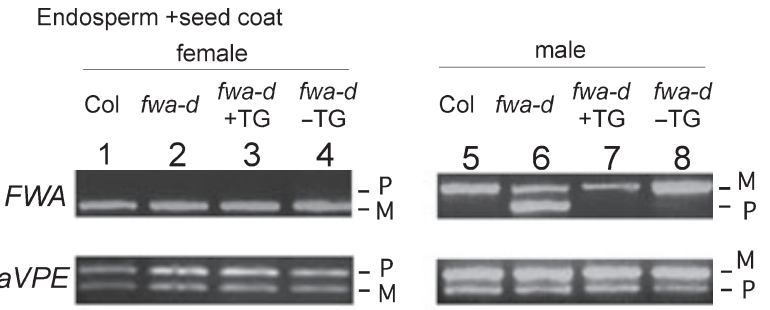

(b) Embryo

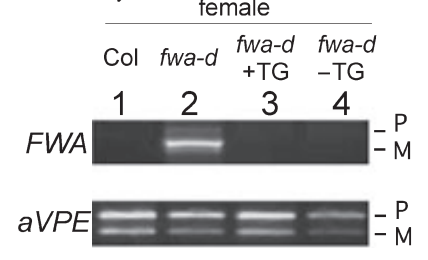

Figure 4. The FWA gene silenced by RdDM was transcribed in endosperm in an imprinted manner.

Wild-type Col (lanes 1 and 5), fwa-d (lanes 2 and 6), fwa- $d$ with the RdDM transgene (lanes 3 and 7) or fwa-d after segregating out of the RdDM transgene (lanes 4 and 8) were used as female (left panel) or male (right panel) parents. All are in the Col background and were crossed to wild-type Ler. In RT-PCR, paternal (P) and maternal (M) transcripts were distinguished by the $\mathrm{Col} /$ Ler polymorphisms as described previously (Kinoshita et al., 2004). RTPCR was performed using total RNA isolated from dissected embryo (b) or endosperm plus seed-coat fraction (a) from developing seeds at the walkingstick embryo stage. aVPE was used as a control with biallelic expression.

wild type, transcription in the endosperm was only from the maternally derived copy; the paternally derived copy remained silent unless it was transmitted directly from the fwa-d allele (Figure 4a, right panel). Transcription was not detectable in the embryo unless it was transmitted directly from the fwa-d allele (Figure 4b).

\section{Discussion}

Using a stable $d d m$ 1-induced late-flowering line ( $f w a-d$ ) and the RdDM procedure, we determined for which region methylation is critical for FWA silencing. FWA became silenced when de novo methylation was induced in tandem direct repeats around the transcription start site. As in the wild type, the newly silenced FWA was transcribed in the endosperm in an imprinted manner. The effect of the RdDM transgene on transcription was heritable, and associated with CG methylation in this region and the presence of corresponding small RNA. These results indicate that methylation of this region is sufficient for proper epigenetic control of the FWA gene. The direct-repeat sequence is structurally similar to the short interspersed nuclear element (SINE) retrotransposon (Lippman et al., 2004; Myouga et al., 2001). Transposon sequences can generally be targets of silencing by DNA methylation (Kato et al., 2003; Lippman et al., 2004; Matzke et al., 1999; Miura et al., 2001; Selker et al., 2003; Singer et al., 2001; Yoder et al., 1997). It would

be interesting to learn whether the SINE-like sequence per se is important, or the repeated feature is necessary for evolution of such an epigenetic control system. In order to understand that, study of the structure and control of the FWA repeats in diverse natural accessions or related Arabidopsis species might be informative.

Interestingly, short direct repeats are often associated with mammalian imprinted genes (Neumann et al., 1995). In addition, a number of epigenetic systems use tandem direct repeats as controlling cis-elements. A direct repeat was identified as the critical element for control of $B$ paramutation in maize, a classical example of epigenetic silencing heritable over multiple plant generations (Stam et al., 2002). Genomes of higher eukaryotes contain many direct repeats, and their epigenetic controls relate to important cellular processes, such as centromere definition and nucleolar dominance (Grewal and Rice, 2004; Pikaard, 2000). The locus responsible for another $d d m$ 1-induced epigenetic variant, $B A L$, is composed of a cluster of diseaseresistance genes organized as both direct and inverted repeats (Stokes et al., 2002). Some endogenous inverted repeat sequences can be the target of de novo methylation (Melquist and Bender, 2003), but the RNAi-based mechanisms might also control silencing of direct repeats (Martienssen, 2003).

The FWA epiallele induced by the $d d m 1$ mutation was stably inherited even in the presence of the wild-type $D D M 1$ copy (Figures S2 and S3). Inheritance of the $d d m$ 1-induced epigenetic changes has also been reported in other sequences (Kakutani et al., 1999; Kato et al., 2004; Lippman et al., 2003; Stokes et al., 2002). Not only the $d d m 1$ but also the met1 mutation induces a late-flowering phenotype (Kankel et al., 2003; Ronemus et al., 1996; Saze et al., 2003). The met1-induced late-flowering trait is also heritable over multiple generations, even after recovery of the MET1 gene function, although a low-frequency reversion was also reported (Kankel et al., 2003; Ronemus et al., 1996). These observations also suggest that methylation of CG sites is necessary for the maintenance of FWA silencing.

In contrast to the inefficient de novo silencing in the ddm1- or met1-induced FWA epiallele, tissue-specific and imprinted FWA expression can be reproduced in the FWAGFP reporter transgene in the first generation (Figure S1). As the FWA transgene in Agrobacterium presumably does not have the proper epigenetic marks, such as histone modifications and DNA methylation, this observation suggests that the transgene acquired the epigenetic marks soon after the transformation event. Although de novo silencing can be induced by the inverted-repeat RNA, transcription of the FWA direct repeat was not sufficient to induce de novo silencing. De novo silencing of direct repeats may require another unknown factor, which can be mimicked by the Agrobacterium-mediated transformation procedure (Chan et al., 2004). A newly integrated DNA may be silenced more 
easily than chromatin already having euchromatic marks. Alternatively, double-strand breaks or other types of DNA damage during the integration process may affect the epigenetic state. Recent findings suggest an overlap in the machinery controlling DNA repair and gene silencing (Elmayan et al., 2005; Takeda et al., 2004). In any case, these features of the integrated genes may induce a defence reaction against integration of foreign DNA such as viruses and transposons (Matzke et al., 1999; Yoder et al., 1997).

\section{Experimental procedures}

\section{Plant materials}

Isolation of $d d m 1$ mutants from Arabidopsis was as reported by Vongs et al. (1993). The ddm1-1 allele in the Columbia (Col) background was used throughout. The $d d m$ 1-1 mutants and wild-type genotypes were distinguished by examining PCR products with primer pairs $5^{\prime}$-ATTTGCTGATGACCAGGTCCT- $3^{\prime}$ and $5^{\prime}$-CATAAACCAATCTCATGAGGC-3', and restriction digestion by $\mathrm{Nsil}$.

\section{Analysis of RNA and genomic DNA}

For FWA expression analysis, RNA was prepared using the RNeasy Plant Mini Kit (Qiagen, Hilden, Germany). RT-PCR was performed using the RETROscript kit (Ambion, St. Austin, TX, USA) or One Step RNA kit (Takara, Ohtsu, Japan). In short, after reverse transcription, cDNA from 50-100-ng input RNA was amplified in 25 PCR cycles and detected by electrophoresis. To detect FWA and control GAPC transcript (Shih et al., 1991), primer pairs 5'-GCTCACTCCAACAGATTCAAGCAG-3' and 5'-GTTGGTAGATGAAAGGGTCGAGAG-3'; and 5'-CACTTGAAGGGTGGTGCCAAG-3' and 5'-CCTGTTGTCGCCAACGAAGTC-3', respectively, were used. Products from genomic DNA and mRNA could be distinguished by size as the intron was included within the amplified region. Southern analysis of genomic DNA was performed as described previously (Miura et al., 2004).

\section{Generation of inverted repeat constructs}

Transgenes expressing double-stranded RNA were created according to the method of Wesley et al. (2001). PCR fragments corresponding to regions $A, B, B 1, B 2$ and $C$ (Figure 2a) were amplified from a BAC clone F11K11, which contains the FWA gene, with the following primer combinations:

A region: 5'-aaaaagcaggctctctcttcctcatctgcgctta- $3^{\prime}$ and $5^{\prime}$-agaaagctgggtcccttaaacaagtaaacactaaaccca-3';

B region: $5^{\prime}$-aaaaagcaggctctgagttatgggccgaagccca- 3 ' and $5^{\prime}$-agaaagctgggtctcgggaaccaaaatcattctct- $3^{\prime}$;

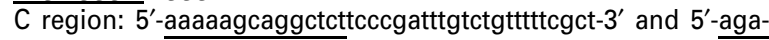
aagctgggtcgcaataacctggacattgcatact- $3^{\prime}$

$\overline{B 1}$ region: 5'-aaaaagcaggctctgagttatgggecgaagccca- $3^{\prime}$ and 5 'agaaagctgggtcctgattgtcagtatcccaca-3';

B2 region: 5'-aaaaagcaggctcttgtgggatactgacaatcag- $3^{\prime}$ and $5^{\prime}$-agaaagctgggtctcgggaaccaaaatcattctct- $3^{\prime}$.

Each of the primers contains the attB1 or attB2 adapter sequence (underlined) at the $5^{\prime}$ end. To introduce whole attB1 and attB2 adapter sequences to the fragments, all PCR fragments were amplified again using primers $5^{\prime}$-ggggacaagtttgtacaaaaagcaggct- $3^{\prime}$ and $5^{\prime}$-ggggaccactttgtacaagaaagctgggt- $3^{\prime}$. The PCR products were then cloned into pHellsgate2 double-stranded RNA producing vector using $B P$ recombinase according to the manufacturer's instructions (Invitrogen, Carlsbad, CA, USA). Plants were transformed via Agrobacterium EHA101 by the floral-dip method (Clough and Bent, 1998). Segregation of the pHellsgate transgene (Figure 3a) was examined by PCR using a primer pair, 35Sf: $5^{\prime}$-cgtaagggatgacgcaca$3^{\prime}$ and OCSr: $5^{\prime}$-aggatctgagctacacatgctca- $3^{\prime}$.

\section{Detection of DNA methylation by the bisulfite method}

Bisulfite sequencing was performed as described by Paulin et al. (1998). After the chemical bisulfite reaction, PCR fragments overlapped to regions $\mathrm{A}-\mathrm{C}$ were amplified with the following primers. For the A region, 5'-aggttytyatyatatayygaaagaatggga- $3^{\prime}$ and $5^{\prime}$ ttraaaccatccatrratrrcctartt-3'; B region, $5^{\prime}$-aaagagttatgggyygaag- $3^{\prime}$ and $5^{\prime}$-crrraaccaaaatcattctctaaaca-3'; C region, $5^{\prime}$-tgtttagagaatgatttggttyyyg- $3^{\prime}$ and $5^{\prime}$-ctaccaacctaarattatttactatttcattccaa- $3^{\prime}$. The amplified PCR fragments were gel-purified and cloned into pT7Blue plasmid (Novagen, San Diego, CA, USA), and then 10-12 independent clones were sequenced. The ASA1 gene (Jeddeloh et al., 1998) was used as a positive control for the bisulfite chemical reaction.

\section{Small RNA Northern analysis}

Small RNA was isolated from mature leaves using the mirVana miRNA isolation kit (Ambion). RNA $(30 \mu \mathrm{g})$ was resolved on denaturing polyacrylamide gels (15\%). Electroblotting was performed as described by Llave et al. (2002). A radiolabeled probe covering the FWA repeat sequence was made by the random priming reaction. Hybridization was performed overnight at $38^{\circ} \mathrm{C}$ using PerfectHyb Plus buffer (Sigma, St. Louis, MO, USA). Blots were washed at $42^{\circ} \mathrm{C}$ in $2 \times \mathrm{SSC}, 0.2 \%$ SDS for 10 and $50 \mathrm{~min}$, and in $0.5 \times \mathrm{SSC}, 0.1 \% \mathrm{SDS}$ for $60 \mathrm{~min}$.

\section{Acknowledgements}

We thank Kazuya Takashiama and Akiko Terui for technical assistance, Hisashi Tamaru for discussion, Tatsuo Kanno and Marjori Matzke for advice on small RNA detection, and Eric Richards for critical comments on the manuscript. We acknowledge The Arabidopsis Biological Resource Center (ABRC) at The Ohio State University for the seeds and BAC clones. Supported by Grant-in-Aid for Creative Scientific Research 14GS0321.

\section{Supplementary Material}

The following supplementary material is available for this article online:

Figure S1. Proper tissue-specific and imprinted silencing of an FWAGFP transgene.

Figure S2. Stable inheritance of the late flowering trait induced by ddm1.

Figure S3. Linkage of the $d d m$ 1-induced late flowering trait to the FWA locus.

Figure S4. siRNA accumulation of the FWA tandem repeats.

Figure S5. Inheritance of hypomethylation in a gene adjacent to FWA.

Appendix S1. Material and methods and references.

This material is available as part of the online article from http:// www.blackwell-synergy.com. 


\section{References}

Cao, X. and Jacobsen, S.E. (2002) Role of the Arabidopsis DRM methyltransferases in de novo DNA methylation and gene silencing. Curr. Biol. 12, 1138-1144.

Chan, S., Zilberman, D., Xie, Z., Johansen, L.K., Carrington, J.C. and Jacobsen, S.E. (2004) RNA silencing genes control de novo DNA methylation. Science, 303, 1336.

Chan, S.W., Henderson, I.R. and Jacobsen, S.E. (2005) Gardening the genome: DNA methylation in Arabidopsis thaliana. Nat. Rev. Genet. 6, 351-360.

Choi, Y., Gehring, M., Johnson, L., Hannon, M., Harada, J.J., Goldberg, R.B., Jacobsen, S.E. and Fischer, R.L. (2002) DEMETER a DNA glycosylase domain protein, is required for endosperm gene imprinting and seed viability in arabidopsis. Cell, 110, 33-42.

Clough, S.J. and Bent, A.F. (1998) Floral dip: a simplified method for Agrobacterium-mediated transformation of Arabidopsis thaliana. Plant J. 16, 735-743.

Elmayan, T., Proux, F. and Vaucheret, H. (2005) Arabidopsis RPA2: a genetic link among transcriptional gene silencing, DNA repair, and DNA replication. Curr. Biol. 8, 1919-1925.

Gehring, M., Huh, J.H., Hsieh, T.F., Penterman, J., Choi, Y., Harada, J.J., Goldberg, R.B., Fischer, R.L. (2006) DEMETER DNA glycosylase establishes MEDEA polycomb gene self-imprinting by allele-specific demethylation. Cell, 124, 495-506.

Grewal, S.I. and Rice, J.C. (2004) Regulation of heterochromatin by histone methylation and small RNAs. Curr. Opin. Cell Biol. 16, 230-238.

Grossniklaus, U., Vielle-Calzada, J.P., Hoeppner, M.A. and Gagliano, W.B. (1998) Maternal control of embryogenesis by MEDEA, a polycomb group gene in Arabidopsis. Science, 280, 446-450.

Hirochika, H., Okamoto, H. and Kakutani, T. (2000) Silencing of retrotransposons in Arabidopsis and reactivation by the $d d m 1$ mutation. Plant Cell, 12, 357-369.

Jeddeloh, J.A., Bender, J. and Richards, E.J. (1998) The DNA methylation locus DDM1 is required for maintenance of gene silencing in Arabidopsis. Genes Dev. 12, 1714-1725.

Kakutani, T. (1997) Genetic characterization of late-flowering traits induced by DNA hypomethylation mutation in Arabidopsis thaliana. Plant J. 12, 1447-1451.

Kakutani, T., Jeddeloh, J., Flowers, S., Munakata, K. and Richards, E. (1996) Developmental abnormalities and epimutations associated with DNA hypomethylation mutations. Proc. Natl Acad. Sci. USA, 93, 12406-12411.

Kakutani, T., Munakata, K., Richards, E. and Hirochika, H. (1999) Meiotically and mitotically stable inheritance of DNA hypomethylation induced by $d d m 1$ mutation of Arabidopsis thaliana. Genetics, 163, 1109-1122.

Kaneda, M., Okano, M., Hata, K., Sado, T., Tsujimoto, N., Li, E. and Sasaki, H. (2004) Essential role for de novo DNA methyltransferase Dnmt3a in paternal and maternal imprinting. Nature, $\mathbf{4 2 9}$, 900-903.

Kankel, M.W., Ramsey, D.E., Stokes, T.L., Flowers, S.K., Haag, J.R., Jeddeloh, J.A., Riddle, N.C., Verbsky, M.L. and Richards, E.J. (2003) Arabidopsis MET1 cytosine methyltransferase mutants. Genetics, 163, 1109-1122.

Kato, M., Miura, A., Bender, J., Jacobsen, S. and Kakutani, T. (2003) Role of CG and non-CG methylation in immobilization of transposons in Arabidopsis. Curr. Biol. 13, 421-426.

Kato, M., Takashima, K. and Kakutani, T. (2004) Epigenetic control of CACTA transposon mobility in Arabidopsis thaliana. Genetics, 168, 961-969.

Kinoshita, T., Yadegari, R., Harada, J.J., Goldberg, R.B. and Fischer, R.L. (1999) Imprinting of the MEDEA polycomb gene in the Arabidopsis endosperm. Plant Cell, 11, 1945-1952.
Kinoshita, T., Miura, A., Choi, Y., Kinoshita, Y., Cao, X., Jacobsen, S.E., Fischer, R.L. and Kakutani, T. (2004) One-way control of FWA imprinting in Arabidopsis endosperm by DNA methylation. Science, 303, 521-523.

Koornneef, M., Hanhart, C. and Veen, J.V.D. (1991) A genetic and physiological analysis of late flowering mutants in Arabidopsis thaliana. Mol. Gen. Genet. 229, 57-66.

Li, E., Beard, C. and Jaenisch, R. (1993) Role for DNA methylation in genomic imprinting. Nature, 366, 362-365.

Lippman, Z., May, B., Yordan, C., Singer, T. and Martienssen, R. (2003) Distinct mechanisms determine transposon inheritance and methylation via small interfering RNA and histone modification. PLoS Biol. 1, E67.

Lippman, Z., Gendrel, A.V., Black, M. et al. (2004) Role of transposable elements in heterochromatin and epigenetic control. Nature, 430, 471-476.

Llave, C., Kasschau, K., Rector, M. and Carrington, J. (2002) Endogenous and silencing-associated small RNAs in plants. Plant Cell, 14, 1605-1619.

Martienssen, R.A. (2003) Maintenance of heterochromatin by RNA interference of tandem repeats. Nat. Genet. 35, 213-214.

Matzke, M.A., Mette, M.F., Aufsatz, W., Jakowisch, J. and Matzke, A.J. (1999) Host defenses to parasitic sequences and the evolution of epigenetic control mechanisms. Genetica, 107, 271-287.

Melquist, S. and Bender, J. (2003) Transcription from an upstream promoter controls methylation signaling from an inverted repeat of endogenous genes in Arabidopsis. Genes Dev. 17, 2036-2047.

Mette, M.F., Aufsatz, W., van der Winden, J., Matzke, M.A. and Matzke, A.J. (2000) Transcriptional silencing and promoter methylation triggered by double-stranded RNA. EMBO J. 19, 5194-5201.

Miura, A., Yonebayashi, S., Watanabe, K., Toyama, T., Shimada, H. and Kakutani, T. (2001) Mobilization of transposons by a mutation abolishing full DNA methylation in Arabidopsis. Nature, 411, 212 214.

Miura, A., Kato, M., Watanabe, K., Kawabe, H., Kotani, H. and Kakutani, T. (2004) Genomic localization of endogenous mobile CACTA family transposons in natural variants of Arabidopsis thaliana. Mol. Genet. Genom. 270, 524-532.

Myouga, F., Tsuchimoto, S., Noma, K., Ohtsubo, H. and Ohtsubo, E. (2001) Identification and structural analysis of SINE elements in the Arabidopsis thaliana genome. Genes Genet. Syst. 74, 169-179.

Neumann, B., Kubicka, P. and Barlow, D.P. (1995) Characteristics of imprinted genes. Nat. Genet. 9, 12-13.

Paulin, R., Grigg, G.W., Davey, M.W. and Piper, A.A. (1998) Urea improves efficiency of bisulphite-mediated sequencing of $5^{\prime}$ methylcytosine in genomic DNA. Nucleic Acids Res. 26, 50095010.

Pikaard, C.S. (2000) The epigenetics of nucleolar dominance. Trends Genet. 16, 495-500.

Rangwala, S.H. and Richards, E.J. (2004) The value-added genome: building and maintaining genomic cytosine methylation landscapes. Curr. Opin. Genet. Dev. 14, 686-691.

Ronemus, M., Galbiati, M., Ticknor, C., Chen, J. and Dellaporta, S. (1996) Demethylation-induced developmental pleiotropy in Arabidopsis. Science, 273, 654-657.

Saze, H., Scheid, O.M. and Paszkowski, J. (2003) Maintenance of CpG methylation is essential for epigenetic inheritance during plant gametogenesis. Nat. Genet. 34, 65-69.

Scott, R.J. and Spielman, M. (2004) Epigenetics: imprinting in plants and mammals - the same but different? Curr. Biol. 14, R201-R203.

Selker, E., Tountas, N., Cross, S., Margolin, B., Murphy, J., Bird, A. and Freitag, M. (2003) The methylated component of the Neurospora crassa genome. Nature, 422, 893-897. 
Shih, M.C., Heinrich, P. and Goodman, H.M. (1991) Cloning and chromosomal mapping of nuclear genes encoding chloroplast and cytosolic glyceraldehyde-3-phosphate-dehydrogenase from Arabidopsis thaliana. Gene, 104, 133-138.

Singer, T., Yordan, C. and Martienssen, R. (2001) Robertson's Mutator transposons in $A$. thaliana are regulated by the chromatin remodeling gene decrease in DNA Methylation (DDM1). Genes Dev. 15, 591-692.

Soppe, W.J., Jacobsen, S.E., Alonso-Blanco, C., Jackson, J.P., Kakutani, T., Koornneef, M. and Peeters, A.J.M. (2000) The late flowering phenotype of fwa mutants is caused by gain-offunction epigenetic alleles of a homeodomain gene. Mol. Cell. 6, 791-802.

Spillane, C., Baroux, C., Escobar-Restrepo, J.M., Page, D.R., Laoueille, S. and Grossniklaus, U. (2004) Transposons and tandem repeats are not involved in the control of genomic imprinting at the MEDEA locus in Arabidopsis. Cold Spring Harb. Symp. Quant. Biol. 69, 465-475.

Stam, M., Belele, C., Dorweiler, J.E. and Chandler, V.L. (2002) Differential chromatin structure within a tandem array $100 \mathrm{~kb}$ upstream of the maize $b 1$ locus is associated with paramutation. Genes Dev. 16, 1906-1918.

Stokes, T.L., Kunkel, B.N. and Richards, E.J. (2002) Epigenetic variation in Arabidopsis disease resistance. Genes Dev. 16, 171 182.
Takeda, S., Tadele, S., Hofmann, I. et al. (2004) BRU1, a novel link between responses to DNA damage and epigenetic gene silencing in Arabidopsis. Genes Dev. 18, 782-793.

Vaucheret, H., Beclin, C., Elmayan, T., Feuerbach, F., Godon, C., Morel, J.B., Mourrain, P., Palauqui, J.C. and Vernhettes, S. (1998) Transgene-induced gene silencing in plants. Plant J. 16, 651-659.

Vielle-Calzada, J.P., Thomas, J., Spillane, C., Coluccio, A., Hoeppner, M.A. and Grossniklaus, U. (1999) Maintenance of genomic imprinting at the Arabidopsis medea locus requires zygotic DDM1 activity. Genes Dev. 13, 2971-2982.

Vongs, A., Kakutani, T., Martienssen, R.A. and Richards, E.J. (1993) Arabidopsis thaliana DNA methylation mutants. Science, 260, 1926-1928.

Wesley, S.V., Helliwell, C.A., Smith, N.A. et al. (2001) Construct design for efficient, effective and high-throughput gene silencing in plants. Plant J. 27, 581-590.

Xiao, W., Gehring, M., Choi, Y., Margossian, L., Pu, H., Harada, J.J., Goldberg, R.B., Pennell, R.I. and Fischer, R.L. (2003) Imprinting of the MEA polycomb gene is controlled by antagonism between MET1 methyltransferase and DME glycosylase. Dev. Cell, 5, 891901.

Yoder, J.A., Walsh, C.P. and Bestor, T.H. (1997) Cytosine methylation and the ecology of intragenomic parasites. Trends Genet. 13, 335-340. 